\title{
Ibuprofen attenuates nephropathy in streptozotocin-induced diabetic rats
}

\author{
YAO-WU LIU, XIA ZHU, YA-QIN CHENG, QIAN LU, FAN ZHANG, HAO GUO and XIAO-XING YIN \\ Jiangsu Key Laboratory of New Drug Research and Clinical Pharmacy, \\ Xuzhou Medical College, Xuzhou, Jiangsu 221004, P.R. China
}

Received March 10, 2015; Accepted January 8, 2016

DOI: $10.3892 / \mathrm{mmr} .2016 .5150$

\begin{abstract}
Ibuprofen, a commonly administered nonsteroidal anti-inflammatory therapeutic agent, is also a partial agonist of peroxisome proliferator-activated receptor $\gamma$ (PPAR $\gamma$ ). The present study investigated the effects of ibuprofen on type 1 diabetic nephropathy (DN) in rats, and the potential mechanisms associated with the activation of PPAR $\gamma$. Diabetic rats were induced through a single intraperitoneal injection of streptozotocin before oral treatment with ibuprofen or pioglitazone for 8 weeks. The 24-h urine collection was performed for measurement of total protein content. The kidney was fixed in $10 \%$ formalin for periodic acid-Schiff and Masson's trichrome staining. Blood and residual kidney tissue samples were collected to measure the associated biochemical parameters. Chronic ibuprofen treatment decreased urinary protein excretion, blood urea nitrogen, glomerular basement membrane thickening and renal fibrosis, which were accompanied by increases in PPAR $\gamma$ protein expression, glutathione (GSH) level, and superoxide dismutase (SOD) activity, decreases in cyclooxygenase 2 and inducible nitric oxide synthase protein expressions, as well as a decreased interleukin $1 \beta$ (IL-1 $\beta$ ) level in the renal cortex of DN rats. Furthermore, the reduced IL-1 $\beta$ level, increased GSH quantities and stronger SOD activity in the rat serum were evaluated in ibuprofen-treated diabetic rats and were compared with untreated diabetic rats. Regarding GSH and IL-1 $\beta$ levels, ibuprofen was identified to be superior to the positive control, pioglitazone, while levels of the other indices were identified to be similar. Thus, ibuprofen was observed to prevent the development of DN, caused by type 1 diabetes, by anti-inflammatory and anti-oxidative action, potentially via PPAR $\gamma$ activation.
\end{abstract}

Correspondence to: Dr Yao-Wu Liu, Jiangsu Key Laboratory of New Drug Research and Clinical Pharmacy, Xuzhou Medical College, 209 Tongshan Road, Xuzhou, Jiangsu 221004, P.R. China

E-mail:w54615@163.com; ywliu@xzhmu.edu.cn

Key words: diabetic nephropathy, ibuprofen, inflammation, oxidative stress, peroxisome proliferator-activated receptor $\gamma$

\section{Introduction}

Diabetic nephropathy (DN), a major complication of type 1 and 2 diabetes, is the most common cause of advanced kidney disease, leading to a high number of diabetes-associated mortalities $(1,2)$. However, investigating the mechanisms underlying this condition is complex due to its complicated pathogenesis, and various or numerous symptoms. At present, DN is widely hypothesized to involve tubulointerstitial fibrosis and glomerulosclerosis, triggered by oxidative stress, inflammation, the activation of the renin-angiotensin-aldosterone system, profibrotic factors, collagen cross-linking and epithelial mesenchymal transition, amongst other causes (3-5). However, to the best of our knowledge, the presence of a possible association between these factors remains to be elucidated.

Peroxisome proliferator-activated receptor $\gamma(\operatorname{PPAR} \gamma)$ is a member of the ligand-activated transcription factors of the nuclear hormone receptor superfamily, and is closely associated with the pathogenesis of numerous diseases, such as diabetes, obesity and inflammatory diseases, including colitis, steatohepatitis, chronic obstructive pulmonary disease and osteoarthritis (6). Regarding the critical role of PPAR $\gamma$ in regulating diverse biological processes, such as lipid metabolism, adipogenesis, and insulin sensitization in diabetes (7), the effects of PPAR $\gamma$ activation on DN are primarily based on type 2 diabetes (8-10). However, PPAR $\gamma$ activation prevents the progression of DN in type 1 diabetes (11). Rosiglitazone and pioglitazone, thiazolidinediones (PPAR $\gamma$ agonists), ameliorate $\mathrm{DN}$ by reducing the expression level of chemerin receptor 23 in the kidney of streptozotocin (STZ)-induced type 1 diabetes in rats (11). In addition, low doses of rosiglitazone halt the progression of experimental nephropathy induced by type 1 diabetes by decreasing renal oxidative stress without effecting lipid alteration in diabetic rats (12). Furthermore, a previous study demonstrated that in Finnish adults with type 1 diabetes, the mortality associated with diabetes was almost entirely confined to those with chronic kidney diseases (13). Additionally, evidence indicates that PPAR $\gamma$ agonists alleviate certain symptoms in various types of renal disease $(10,14,15)$. Together, these studies suggested PPAR $\gamma$ activation as an essential therapeutic strategy for kidney diseases caused by type 2 and 1 diabetes.

Previous studies show that the pathogenesis of type $1 \mathrm{DN}$ is associated with inflammation and oxidative stress (16-18). 
Recently, it was proposed that PPAR $\gamma$ agonists exert independent actions on the kidney functions, which may assist with preventing diabetic kidney disease, including important effects on inhibition of inflammation $(17,19)$, oxidative stress $(18,20)$, and advanced glycation end products and their receptor interaction $(19,20)$. Therefore, PPAR $\gamma$ may be associated with oxidative stress, inflammation and other factors during the pathogenesis of DN. In addition, suppression of cyclooxygenase 2 (COX-2)-mediated prostaglandin E2 production decreases inflammation and albuminuria in STZ-induced type 1 diabetic mice (21). Notably, previous studies show that, in addition to inhibition of COX-2, certain nonsteroidal anti-inflammatory drugs (NSAIDs), such as ibuprofen and indomethacin, partially activate PPAR $\gamma$ (22). Thus, due to this double action on inflammation, such NSAIDs may be more efficacious than PPAR $\gamma$ agonists, thiazolidinediones, in the treatment of diabetic kidney diseases.

Based on the above-mentioned findings, the present study aimed to investigate the effects of ibuprofen, a partial agonist of PPAR $\gamma$, (a widely used NSAID with fewer and lighter side effects) on DN, inflammatory response and oxidative stress in STZ-induced type 1 diabetes in rats. The effects of ibuprofen were compared with those of thiazolidinediones, and the thiazolidinedione, pioglitazone served as a positive control.

\section{Materials and methods}

Animals. Male Sprague Dawley rats (age, 10 weeks, $\mathrm{n}=40$ ) were bred at the Xuzhou Medical College Experimental Animal Centre (Xuzhou, China) . Rats were housed in cages (5 rats per cage, 2 cages per group) at $50 \pm 10 \%$ humidity (temperature, $24 \pm 1^{\circ} \mathrm{C}$ ) under a 12 -h light/dark cycle, with free access to water and rodent chow. All animal experiments were approved by the Animal Ethics Committee of Xuzhou Medical College before being performed according to the Guidelines for Ethical Conduct in the Care and Use of Animals (23). Every effort was made to minimise stress to the animals.

Experimental design. The rats were fasted for $12 \mathrm{~h}$ and subjected to a single intraperitoneal injection of $60 \mathrm{mg} / \mathrm{kg} \mathrm{STZ}$ (Sigma-Aldrich, St. Louis, MO, USA), freshly dissolved in $0.1 \mathrm{~mol} / \mathrm{l}$ sodium citrate buffer $(\mathrm{pH} 4.5$; Sinopharm Chemical Reagent Co., Ltd., Shanghai, China). Age-matched healthy rats $(n=10)$ received sodium citrate buffer alone. The development of diabetes was assessed in accordance with non-fasting blood glucose (nFBG) levels using a reagent kit (Jiancheng Bioengineering Institute, Nanjing, China). Subsequent to 7 days of STZ treatment, diabetic rats (those with nFBG $\geq 16.7 \mathrm{mmol} / \mathrm{l}$ ) (24) were successfully obtained, and randomly divided into three groups $(n=10)$ as follows: Diabetic (DM); ibuprofen-treated (DM + IB; $40 \mathrm{mg} / \mathrm{kg}$ ); pioglitazone-treated $(\mathrm{DM}+\mathrm{PI} ; 25 \mathrm{mg} / \mathrm{kg})$. The administered volume of ibuprofen (purity >99\%; Huayida Technology Co., Ltd., Wuhan, China) and pioglitazone (purity $>99 \%$; Zhongke Yitong Chemical Co., Ltd., Jinan, China) was $10 \mathrm{ml} / \mathrm{kg}$, with the concentrations of 4 and $2.5 \mathrm{mg} / \mathrm{ml}$, respectively, prepared in $1 \% \mathrm{w} / \mathrm{v}$ sodium carboxymethyl cellulose (Sinopharm Chemical Reagent Co., Ltd.). Ibuprofen and pioglitazone were administered orally once per day for 8 weeks. The rats were weighed weekly and underwent blood glucose tests, for which the nFBG levels were measured using a reagent kit (Jiancheng Bioengineering Institute), according to the manufacturer's instructions, and ultraviolet-visible (UV-Vis) spectrophotometry (722N UV-Vis spectrophotometer; INESA Analytical Instrument Co., Ltd., Shanghai, China). Eight weeks later, the rats were placed in metabolic cages for 24-h urine collection and consequent albuminuria measurement prior to being sacrificed under ethyl ether (Sinopharm Chemical Reagent Co., Ltd.) anesthesia, using cotton balls soaked with ether. The albuminuria levels were measured using a urine protein test kit (Jiancheng Bioengineering Institute; cat. no. C035-2), according to the manufacturer's instructions, and UV-Vis spectrophotometry. Their blood samples $(\sim 3 \mathrm{ml})$ were collected via femoral vein bleeding, and the serum was collected following centrifugation at $4^{\circ} \mathrm{C}$ at $1,500 \mathrm{x} \mathrm{g}$ for $10 \mathrm{~min}$, for approximately $1 \mathrm{ml}$ of blood. Bilateral kidneys were removed and the left kidney was decapsulated and fixed in $4 \%$ buffered formalin (Sinopharm Chemical Reagent Co., Ltd.) for $24 \mathrm{~h}$ prior to paraffin (Beyotime Institute of Biotechnology, Nantong, China) embedding. In addition, the renal cortex was rapidly isolated. The samples were stored at $-70^{\circ} \mathrm{C}$ prior to use.

Renal function assessment. Renal function can be evaluated through measurement of urinary protein and blood urea nitrogen (BUN). Excretion of urinary protein was quantified using a urine protein test kit (Jiancheng Bioengineering Institute) through the Coomassie (Beyotime Institute of Biotechnology) brilliant blue method, while BUN was examined using a BUN assay kit (Jiancheng Bioengineering Institute) according to a diacetyl oxime colorimetric method (25).

Renal pathological changes by periodic acid-Schiff (PAS) staining and Masson's trichrome staining. Renal PAS staining (Sigma-Aldrich) and Masson's trichrome (Shanghai Yuanye Bio-Technology Co., Ltd., Shanghai, China) staining were performed, as previously described (26). Kidney tissue samples were fixed in a $10 \%$ buffered formalin solution and embedded in paraffin for histological analysis. The $3-\mu \mathrm{m}$ thick paraffin sections were dewaxed through a series of graded ethanol baths, to displace the water, and subsequently infiltrated with water. The sections were stained with PAS or Masson's trichrome, cleared in xylene (Sinopharm Chemical Reagent Co., Ltd.) and mounted with neutral balsam (Sinopharm Chemical Reagent Co., Ltd.) prior to examination under an Olympus BX50 microscope (Olympus Corporation, Tokyo, Japan). The three typical views of each imperfect section were analyzed. Linear measurements were obtained using an image analysis system (Image-Pro Plus 4.0; Media Cybernetics, Inc., Silver Spring, MD, USA).

PPAR $\gamma$ protein expression in rat kidneys by immunohistochemistry. Kidney sections $(4 \mu \mathrm{m})$ were deparaffinized and endogenous peroxidase was blocked by the addition of $3 \% \mathrm{H}_{2} \mathrm{O}_{2}$ (Zhongshan Golden Bridge Biotech Co., Ltd.; OriGene Technologies, Inc., Beijing, China) for $10 \mathrm{~min}$. The sections were incubated overnight at $4{ }^{\circ} \mathrm{C}$ with a rabbit polyclonal anti-PPAR $\gamma$ antibody (1:1,000; Bioworld Technology, Inc., St. Louis Park, MN, USA; cat. no. AP0688), then a polymer helper for $20 \mathrm{~min}$, followed by a polyclonal horseradish-peroxidase-conjugated goat anti-rabbit immunoglobulin $\mathrm{G}$ antibody 
(Zhongshan Golden Bridge Biotech Co., Ltd.; OriGene Technologies, Inc.; cat. no. PV-9001) at room temperature for $20 \mathrm{~min}$. The peroxidase was visualized through the addition of 3,3'-diaminobenzidine (Zhongshan Golden Bridge Biotech Co., Ltd.; OriGene Technologies, Inc.) in the dark for $3 \mathrm{~min}$. The sections were counterstained with hematoxylin (Beyotime Institute of Biotechnology), dehydrated, and observed under an Olympus CX22 light microscope (Olympus Corporation). A minimum of 3 relatively intact kidney tissue sections were selected at random and further incubated with either a primary or a secondary antibody to determine the binding specificity of PPAR $\gamma$, without any positive staining. Five sections were analyzed for each rat, and 10 images were obtained from a randomly selected site per slide. The optical density (OD) of PPAR $\gamma$ immunostaining in the cell nucleus was quantified using Image-Pro Plus 4.0 software.

Protein expression of $\mathrm{COX}-2$ and inducible nitric oxide synthase (iNOS) in the renal cortex by western blotting. The preserved renal cortex was weighed and homogenized using an electronic tissue homogenizer (GF-1; Kylin-Bell Lab Instruments Co., Ltd., Haimen, China) in 10 vol (w/v) Tris-buffered saline $(50 \mathrm{mmol} / \mathrm{l}$; $\mathrm{pH}$ 7.4; Beyotime Institute of Biotechnology) containing $0.6 \mathrm{mmol} / 1$ phenylmethylsulphonyl fluoride (Beyotime Institute of Biotechnology), $1 \mathrm{mmol} / 1$ $\mathrm{Na}_{3} \mathrm{VO}_{4}$ (Sangon Biotech Co. Ltd., Shanghai, China) and $50 \mathrm{mmol} / 1 \mathrm{NaF}$ (Sinopharm Chemical Reagent Co., Ltd.) in an ice bath. The resulting homogenates were maintained at $4^{\circ} \mathrm{C}$ for $\geq 60 \mathrm{~min}$ prior to centrifugation at $4^{\circ} \mathrm{C}$ for $15 \mathrm{~min}$ at $10,000 \mathrm{x} g$ to obtain the supernatant for western blot analysis. Protein concentrations in the supernatant were determined using the bicinchoninic acid protein assay kit (Beyotime Institute of Biotechnology).

Protein samples $(80 \mu \mathrm{g})$ were separated by sodium dodecyl sulphate-polyacrylamide gel electrophoresis (10\% gel; run at 100 volts for $80 \mathrm{~min}$; Beyotime Institute of Biotechnology) and transferred to BioTrace nitrocellulose membranes (Merck Millipore Ireland BV, Co Cork, Ireland). The membranes were blocked with $5 \%$ blocking buffer (Beyotime Institute of Biotechnology) for $120 \mathrm{~min}$ and incubated overnight at $4^{\circ} \mathrm{C}$ with primary antibodies, including rabbit polyclonal anti-COX-2 (1:1,000; ProteinTech Group, Inc., Chicago, IL, USA; cat. .no. 55070-1-AP), rabbit polyclonal anti-iNOS (1:100; Abcam, Inc., Cambridge, UK; cat. .no. ab3523) and rabbit polyclonal anti- $\beta$-actin antibody $(1: 2,000$, Bioworld Technology, Inc.; cat. no. AP0060). The blots were detected using alkaline phosphatase-conjugated affinipure goat anti-rabbit secondary antibody (1:1,000; Zhongshan Golden Bridge Biotech Co., Ltd.; OriGene Technologies, Inc.; cat. no. ZB-2308). The membranes were exposed to a 5-bromo-4-chloro-3-indolyl-phosphate/nitro blue tetrazolium (NBT) alkaline phosphatase color developing reagent (Beyotime Institute of Biotechnology) for $15 \mathrm{~min}$. Signal densities on the blots were measured with Image J software, version 1.48u (http://imagej.nih. gov/ij/) and normalized against rabbit anti- $\beta$-actin (Bioworld Technology Inc.), which served as an internal control $\left(\mathrm{OD}_{\text {protein }} / \mathrm{OD}_{\text {internal control }}\right)$.

Determination of interleukin-1 $\beta(I L-1 \beta)$ levels in the rat kidney and serum by enzyme linked immunosorbent assay (ELISA.
The level of IL-1 $\beta$ in the rat renal cortex and serum was estimated using a commercial ELISA kit (ExCell Biology, Inc., Shanghai, China) according to the manufacturer's instructions.

Assay of superoxide dismutase (SOD) activity and reduced glutathione (GSH) level in the rat kidney and serum. SOD activity was measured using the Total Superoxide Dismutase (T-SOD) assay kit (Hydroxylamine method; Jiancheng Bioengineering Institute; cat. no. A001-1) according to the manufacturer's instructions. Briefly, tissue was prepared in a $10 \%$ solution ( $100 \mathrm{mg}$ tissue in $1,000 \mu \mathrm{l}$ normal saline) and mixed with the reagents of the kit using a vortex mixer. The solution was incubated at $37^{\circ} \mathrm{C}$ for $40 \mathrm{~min}$ prior to the addition of the chromogenic agent. Samples were incubated at room temperature for $10 \mathrm{~min}$ and the OD was then determined at a wavelength of $550 \mathrm{~nm}$. SOD activity (U/mg prot) was calculated as follows: $\mathrm{SOD}_{\text {activity }}=\left(\mathrm{OD}_{\text {control }}-\mathrm{OD}_{\text {test }}\right) / \mathrm{OD}_{\text {control }} \div 50 \% \times \mathrm{TRV} / \mathrm{SV} \div \mathrm{PrCn}$, where TRV is the total reaction volume $(\mathrm{ml}), \mathrm{SV}$ is the sample volume $(\mathrm{ml})$ and $\mathrm{PrCn}$ is the protein concentration of sample (mg prot $/ \mathrm{ml}$ ). The reduced glutathione (GSH) assay (Jiancheng Bioengineering Institute; cat. no. A006-2) was performed according to a previously reported spectrophotometric method (27), using the $722 \mathrm{~N} \mathrm{UV-Vis} \mathrm{spectrophotometer.} \mathrm{One}$ unit of SOD was defined as the quantity of enzyme causing $50 \%$ inhibitory rates of NBT reduction. The tissue GSH level was expressed as nanomoles of GSH per milligram protein, and the serum GSH level was expressed as micromoles of GSH per liter.

Statistical analysis. The results are expressed as means \pm standard deviation. Intergroup variation was measured by one-way analysis of variance followed by Tukey's test. The analysis was performed using SPSS Statistical Software, version 13.0 (SPSS, Inc., Chicago, IL, USA) and $\mathrm{P}<0.05$ was considered to indicate a statistically significant difference.

\section{Results}

Effects of ibuprofen on $n F B G$ and the body weight of diabetic rats. The serum glucose levels of experimental rats were measured at weeks 0 and 8 weeks after treatment with $40 \mathrm{mg} / \mathrm{kg}$ ibuprofen and $25 \mathrm{mg} / \mathrm{kg}$ pioglitazone (the positive control). Compared with the age-matched healthy rats, higher nFBG levels were observed in the diabetic rats throughout the treatment $(\mathrm{P}<0.01)$. However, $\mathrm{nFBG}$ remained at a high level in diabetic rats treated with either ibuprofen or pioglitazone, without any significant difference between the treated and untreated groups (Table I). Furthermore, the body weights of the experimental rats were measured from week 1 to week 8 following treatment with $40 \mathrm{mg} / \mathrm{kg}$ ibuprofen and $25 \mathrm{mg} / \mathrm{kg}$ pioglitazone. Diabetic rats continually demonstrated reduced body weights in comparison to the age-matched healthy rats following the $\mathrm{STZ}$ injection $(\mathrm{P}<0.01)$. Ibuprofen or pioglitazone treatment produced no obvious effect on the body weights of diabetic rats (Table II).

Effects of ibuprofen on renal dysfunction in diabetic rats. Fig. 1 illustrates the effects of ibuprofen and the positive control, pioglitazone on rat serum BUN (Fig. 1A), and urinary protein excretion (Fig. 1B) in the healthy and experimental rats. 
Significant increases were observed in urinary protein excretion and serum BUN level in the diabetic rats compared with the healthy rats $(\mathrm{P}<0.01)$. Chronic treatment with ibuprofen significantly attenuated the damage resulting from renal function, as evidenced by decreases in urinary protein excretion $(\mathrm{P}<0.01)$ and serum BUN level $(\mathrm{P}<0.05)$ of diabetic rats. This finding was also observed in pioglitazone-treated diabetic rats (Fig. 1).

Effects of ibuprofen on glomerular basement membrane thickening and renal fibrosis in the kidney of diabetic rats. Glomerular basement membrane thickening is a pathological feature of DN. These changes were present in the kidneys of the healthy (Fig. 2Aa) and experimental (Fig. 2Ab-d) rats, and were visualized by PAS staining. Diabetic rats demonstrated obvious glomerular basement membrane thickening, which was characterized by a significant increase in the PAS-stained positive area, when compared with that of the healthy rats $(\mathrm{P}<0.01$; Fig. 2B). However, treatment of the diabetic rats with ibuprofen or pioglitazone significantly reduced glomerular basement membrane thickening $(\mathrm{P}<0.01$; Fig. $2 \mathrm{~B})$.

Renal fibrosis is another pathological feature of $\mathrm{DN}$. Fig. 3 demonstrates the renal fibrosis of rats from the healthy (Fig. 3Aa) and experimental (Fig. 3Ab-d) groups using Masson's trichrome staining. Collagen fibers were stained blue, muscle fiber cytoplasm was stained red and nuclei were stained brown. The results reveal a marginal quantity of collagen fiber deposition in the healthy rats (Fig. 3B), but a significant increase in tubulointerstitial collagen in the diabetic rats $(\mathrm{P}<0.01$; Fig. $3 \mathrm{~B})$. Diabetic rats that received ibuprofen or pioglitazone treatment exhibited less tubulointerstitial collagen when compared with the untreated diabetic rats $(\mathrm{P}<0.05$; Fig. $3 \mathrm{~B})$.

Effects of ibuprofen on PPAR $\gamma$ protein expression in the kidney of diabetic rats. Ibuprofen and pioglitazone activate PPAR $\gamma$. To examine the effects of the two therapeutic agents on activation of PPAR $\gamma$, the activated form of PPAR $\gamma$ was identified in the kidney using immunohistochemistry (Fig. 4). The data indicates that the protein expression level of PPAR $\gamma$ in the cell nuclei was markedly reduced in the kidney of diabetic rats when compared with that of healthy rats $(\mathrm{P}<0.01$; Fig. $4 \mathrm{~B})$, while chronic ibuprofen treatment prevented this reduction, with no significant difference when compared with pioglitazone (Fig. 4B).

Effects of ibuprofen on inflammatory responses in diabetic rats. The DN rats displayed marked inflammatory responses in the kidney; protein expressions of COX-2 and iNOS were significantly increased in the renal cortex of diabetic rats, compared with those of the healthy rats $(\mathrm{P}<0.01$ and $\mathrm{P}<0.05$, respectively). Chronic treatment with ibuprofen prevented the increase of COX-2 and iNOS, while treatment with pioglitazone significantly decreased the protein expression of iNOS ( $\mathrm{P}<0.01$ vs. DM), but not that of COX-2 (Fig. 5A). Furthermore, the level of IL-1 $\beta$, an important pro-inflammatory cytokine, was markedly elevated in the renal cortex and serum $(\mathrm{P}<0.01)$ of $\mathrm{DN}$ rats compared with that of healthy rats, which was significantly attenuated following ibuprofen treatment $(\mathrm{P}<0.05)$. By contrast, pioglitazone treatment prevented the elevated IL-1 $\beta$ level in the serum of $\mathrm{DN}$ rats more effectively $(\mathrm{P}<0.05)$ than ibuprofen
Table I. Effects of IB on nFBG of streptozotocin-induced diabetic rats before and after treatment with IB.

\begin{tabular}{llcc}
\hline & & \multicolumn{2}{c}{$\mathrm{nFBG}(\mathrm{mmol} / \mathrm{l})$} \\
\cline { 3 - 4 } Group & $\mathrm{n}$ & Before treatment & After treatment \\
\hline Control & 10 & $6.2 \pm 0.5$ & $4.7 \pm 0.4$ \\
DM & $9-10$ & $36.8 \pm 5.4^{\mathrm{a}}$ & $32.4 \pm 4.3^{\mathrm{a}}$ \\
$\mathrm{DM}+$ PI & 10 & $36.6 \pm 4.6$ & $29.9 \pm 4.2$ \\
DM + IB & $9-10$ & $37.1 \pm 5.7$ & $27.1 \pm 6.1$ \\
\hline
\end{tabular}

Data are presented as means \pm standard deviation. ${ }^{\text {a }}<0.01$ vs. control. nFBG, non-fasting blood glucose; DM, diabetic group; PI, pioglitazone; IB, ibuprofen.

treatment, and although PI treatment decreased the IL-1 $\beta$ level in the renal cortex of DN rats, it exhibited a weaker effect than ibuprofen treatment (Fig. 5B and C).

Effects of ibuprofen on anti-oxidative function in diabetic rats. In addition to inflammatory responses, oxidative stress damage in DN rats was observed. Diabetes induced significant decreases in SOD activity (Fig. 6) and GSH level (Fig. 7) in the renal cortex of rats compared with healthy rats $(\mathrm{P}<0.01$ and $\mathrm{P}<0.05$, respectively), which were alleviated by chronic treatment with ibuprofen and pioglitazone (Figs. 6A and 7A), with no marked difference between the two. Furthermore, SOD activity and GSH level were markedly reduced in the serum of diabetic rats $(\mathrm{P}<0.01)$, while chronic treatment with ibuprofen enhanced SOD activity and the GSH level $(\mathrm{P}<0.01$; Figs. 6B and 7B). Pioglitazone treatment had a similar effect on SOD activity, but a weaker effect on GSH level $(\mathrm{P}<0.05)$ when compared with ibuprofen treatment (Figs. 6B and 7B).

\section{Discussion}

Pathogenesis of DN is complex and multifactorial, involving different cell types, molecules, and factors, in particular oxidative stress and inflammatory cytokines (16). The present study demonstrated significant elevations of urinary protein excretion and BUN level, as well as glomerular basement membrane thickening and renal fibrosis in type 1 diabetic rats over a nine-week period. Diabetes produced large quantities of COX-2, iNOS, and IL- $1 \beta$ protein, but small quantities of PPAR $\gamma$ protein, SOD activity, and GSH levels in the kidney of rats. In addition, high IL- $1 \beta$ levels, and low SOD activity and GSH levels were observed in the serum of diabetic rats. However, the majority of the changes were markedly attenuated by treatment with ibuprofen and pioglitazone, which served as a positive control. These data indicated that ibuprofen prevents the progression of DN in an experimental rat model of type 1 diabetes, via suppression of inflammatory and oxidative damage, potentially through PPAR $\gamma$ activation.

Chronic inflammation is significant in the development of diabetes and its late complications, including DN (28-30). Inflammatory markers are well known to be associated with the development of renal disease in diabetes (31). Anti-inflammatory therapeutic agents have been reported to prevent renal 
Table II. Effects of IB on the body weight of streptozotocin-induced diabetic rats after treatment.

\begin{tabular}{llllllllll}
\hline & \multicolumn{7}{c}{ Body weight $(\mathrm{g})$} \\
\cline { 2 - 9 } Group & Week 0 & Week 1 & Week 2 & Week 3 & Week 4 & Week 5 & Week 6 & Week 7 & Week 8 \\
\hline Control & $245 \pm 4$ & $275 \pm 12$ & $312 \pm 14$ & $337 \pm 15$ & $356 \pm 16$ & $382 \pm 19$ & $393 \pm 19$ & $409 \pm 18$ & $391 \pm 15$ \\
DM & $185 \pm 17^{\text {a }}$ & $186 \pm 25^{\text {a }}$ & $173 \pm 29^{\text {a }}$ & $175 \pm 27^{\text {a }}$ & $174 \pm 32^{\text {a }}$ & $173 \pm 32^{\text {a }}$ & $166 \pm 31^{\text {a }}$ & $168 \pm 38^{\text {a }}$ & $158 \pm 34^{\text {a }}$ \\
DM + PI & $190 \pm 18$ & $180 \pm 28$ & $181 \pm 27$ & $180 \pm 28$ & $183 \pm 33$ & $190 \pm 39$ & $187 \pm 36$ & $180 \pm 37$ & $172 \pm 32$ \\
DM + IB & $183 \pm 24$ & $182 \pm 27$ & $164 \pm 21$ & $175 \pm 32$ & $174 \pm 39$ & $178 \pm 44$ & $163 \pm 41$ & $168 \pm 44$ & $168 \pm 45$
\end{tabular}

Data are presented as means \pm standard deviation; $n=9-10 .{ }^{a} \mathrm{P}<0.01$ vs. control. DM, diabetic group; PI, pioglitazone; IB, ibuprofen.

A

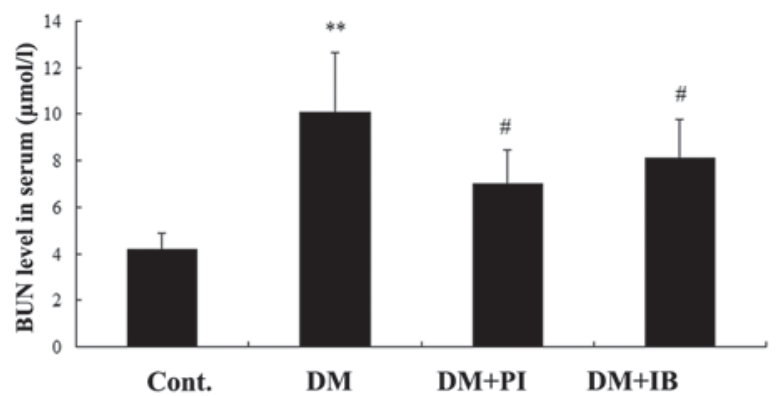

B

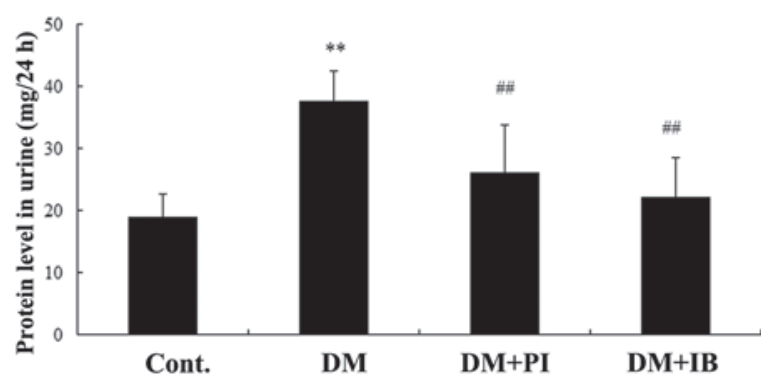

Figure 1. Effects of IB on (A) BUN and (B) urinary protein excretion. Cont., $\mathrm{DM}, \mathrm{DM}+\mathrm{PI}$, and $\mathrm{DM}+\mathrm{IB}$ represent healthy rats, diabetic rats, and diabetic rats treated with PI $(25 \mathrm{mg} / \mathrm{kg})$ and IB $(40 \mathrm{mg} / \mathrm{kg})$, respectively. Data are presented as means \pm standard deviation; $\mathrm{n}=7$. ${ }^{* *} \mathrm{P}<0.01$ vs. Cont.; ${ }^{\prime} \mathrm{P}<0.05$ and ${ }^{\# \#} \mathrm{P}<0.01$ vs. DM. BUN, blood urea nitrogen; Cont., control; DM, diabetic group; PI, pioglitazone; IB, ibuprofen.

injury in diabetic rats by reducing macrophage infiltration and the protein expression of transforming growth factor- $\beta$, type IV collagen and intercellular adhesion molecule 1 in renal cells (32). Furthermore, chronic COX inhibition by NSAIDs (non-selective COX inhibitor, ibuprofen and selective COX-2 inhibitor, NS-398) reduced diabetes-induced hyperfiltration, proteinuria and fibrosis in the kidney of Akita DN mice (33); with the effects of ibuprofen observed to be similar to, if not more beneficial than, COX-2 inhibition by NS-398. In the present study, chronic ibuprofen treatment at a low dose $(40 \mathrm{mg} / \mathrm{kg})$ attenuated proteinuria and renal fibrosis of diabetic rats, which was coupled with the reduced protein expression of COX-2 and iNOS (common inducible proteins in the presence of inflammation) in the renal cortex. In a previous study, IL-1 was hypothesized to increase vascular permeability, mesangial cell proliferation and extracellular matrix deposition, as well as glomerular basement membrane thickening (34). The present study
A a
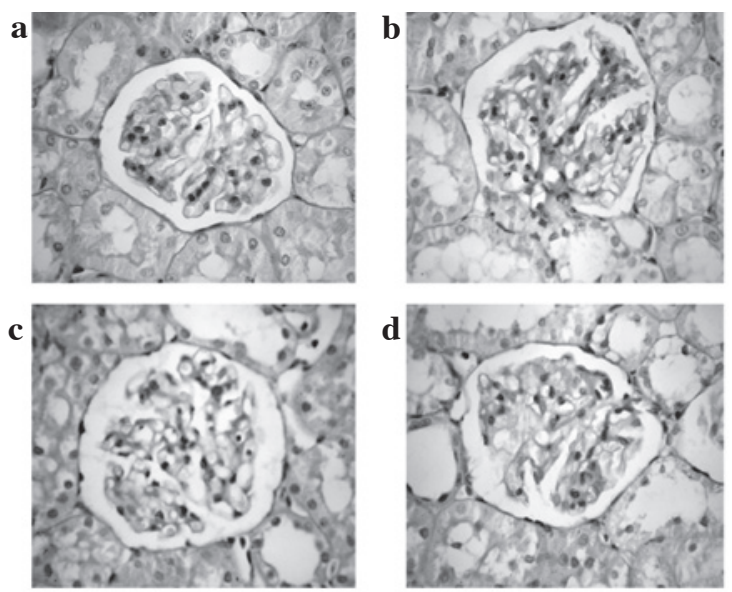

B

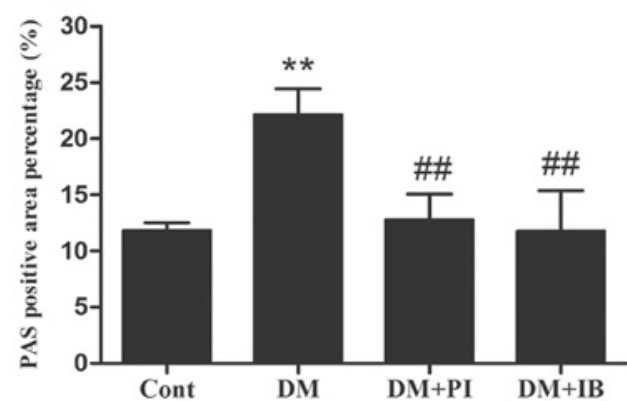

Figure 2. Effects of IB on glomerular basement membrane thickening in rat kidneys. Glomerular basement membrane thickening was examined by PAS staining. (A) Typical staining images (magnification, $x 400$ ) of (a) Cont., (b) DM, (c) DM + PI and (d) DM + IB, and (B) quantitative analysis. The Cont., DM, DM + PI, and DM + IB groups represent healthy rats, and diabetic rats, diabetic rats treated with PI $(25 \mathrm{mg} / \mathrm{kg})$ and IB $(40 \mathrm{mg} / \mathrm{kg})$, respectively. Data are presented as means \pm standard deviation; $n=3$. ${ }^{* *} \mathrm{P}<0.01$ vs. Cont.; ${ }^{\# \#} \mathrm{P}<0.01$ vs. DM. PAS, Periodic acid-Schiff; Cont., control; DM, diabetic group; PI, pioglitazone; IB, ibuprofen.

identified that glomerular basement membrane thickening occurred in the kidney of diabetic rats, with increased IL-1 $\beta$ levels observed in the serum and renal cortex. However, ibuprofen prevented glomerular basement membrane thickening, and decreased the IL-1 $\beta$ level in the blood and kidney. The above-mentioned studies and the results of the present study suggest that anti-inflammatory therapy alleviates functional and morphological impairments of diabetic animals. A previous study demonstrated that PPAR $\gamma$ agonists attenuated renal injury and inflammation in a mouse model of unilateral ureteral obstruction (35). Ohga et al (6) reported that 
A
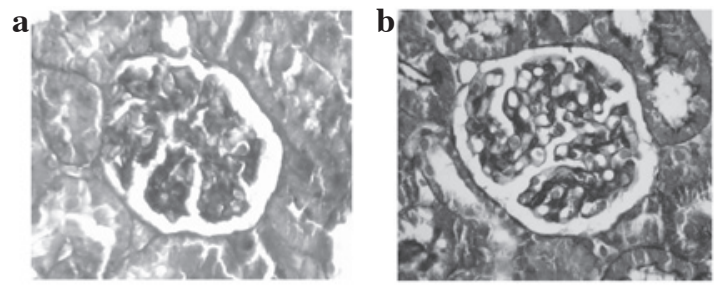

c
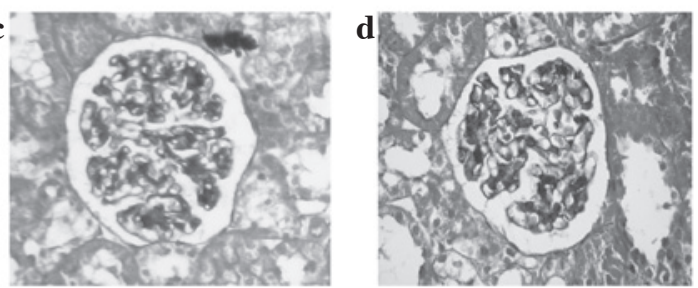

B

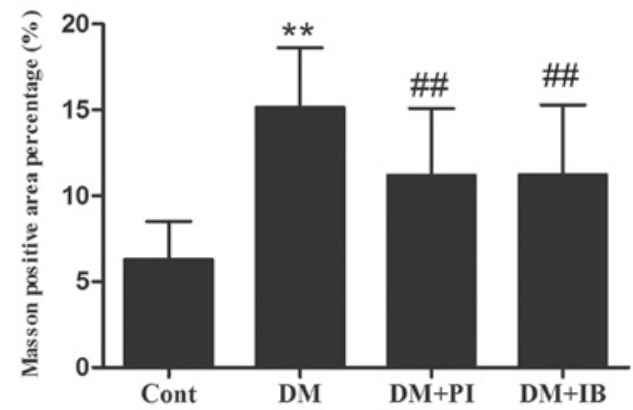

Figure 3. Effects of IB on rat kidney renal fibrosis, which was assessed by Masson's trichrome staining. (A) Typical staining images (magnification, x400) of (a) Cont., (b) DM, (c) DM + PI and (d) DM + IB, and (B) quantitative analysis. The Cont., DM, DM + PI, and DM + IB groups represent healthy rats, diabetic rats, and diabetic rats treated with PI $(25 \mathrm{mg} / \mathrm{kg})$ and IB $(40 \mathrm{mg} / \mathrm{kg})$, respectively. Data are presented as means \pm standard deviation ( $\mathrm{n}=3$ ). ${ }^{* *} \mathrm{P}<0.01$ vs. Cont.; ${ }^{\#} \mathrm{P}<0.05$ vs. DM. Cont., control; DM, diabetic group; PI, pioglitazone; IB, ibuprofen.

pioglitazone ameliorated renal injury through the inhibition of nuclear factor $\kappa$-light-chain-enhancer of activated B cells activation, intercellular adhesion molecule 1 expression and macrophage infiltration in STZ-induced diabetic rats. The present findings showed that ibuprofen, a typical NSAID and partial agonist of PPAR $\gamma$, decreased the level of inflammatory markers in the renal cortex, and elevated the activated PPAR $\gamma$ level in the kidneys of diabetic rats. These results demonstrated that the anti-inflammatory effect, possibly via PPAR $\gamma$ activation, was responsible for the alleviation of DN by ibuprofen in type 1 diabetes.

In addition to chronic inflammation, oxidative stress was hypothesized to be vital in the pathological process of chronic diabetic complications, including DN (36,37). A unifying hypothesis has been proposed, where over-production of reactive oxygen species in mitochondria, in response to chronic hyperglycemia, may be the key initiator of various pathogenic pathways in diabetic complications (38). Gumieniczek (39) reported that GSH reductase activity and the GSH level were diminished in the kidney of alloxan-induced diabetes in rabbits, and pioglitazone restored them to the control values. In the current study, SOD activity and GSH level, two important endogenous antioxidants, were significantly declined in the renal cortex and serum of STZ-induced diabetes in rats. Furthermore, oxidative stress and renal fibrosis in the kidney of STZ-induced diabetes in mice have
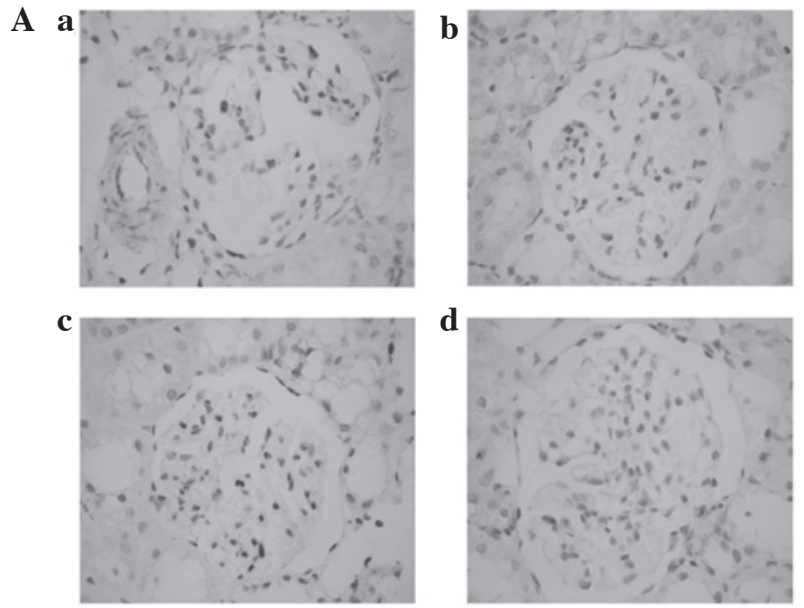

B

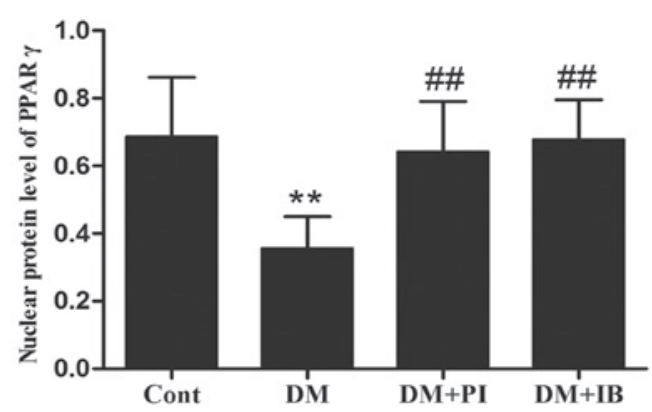

Figure 4. Effects of IB on the activated form of PPAR $\gamma$ in rat kidneys. Protein expression of PPAR $\gamma$ in the nucleus was assayed by immunohistochemistry. (A) Typical staining images (magnification, x400) of (a) Cont., (b) DM, (c) DM + PI and (d) DM + IB, and (B) quantitative analysis. The Cont. $\mathrm{DM}, \mathrm{DM}+\mathrm{PI}$, and DM + IB groups represent healthy rats, diabetic rats, and diabetic rats treated with PI $(25 \mathrm{mg} / \mathrm{kg})$ and IB $(40 \mathrm{mg} / \mathrm{kg})$, respectively. Data are presented as means \pm standard deviation; $\mathrm{n}=3 .{ }^{* *} \mathrm{P}<0.01$ vs. Cont.; ${ }^{\#} \mathrm{P}<0.01$ vs. DM. PPAR $\gamma$, peroxisome proliferator-activated receptor $\gamma$; Cont., control; DM, diabetic group; PI, pioglitazone; IB, ibuprofen.

previously been associated with decreased protein expression of PPAR $\gamma$ and its coactivator PGC- $1 \alpha$, which may be attenuated by telmisartan (18). The present findings indicated that ibuprofen and pioglitazone relieved the oxidative damage in the kidney of diabetic rats, in combination with a marked increase in protein expression of nuclear PPAR $\gamma$ in the kidney. Notably, a previous study indicated that ibuprofen disrupted the signaling cascades that lead to microglial nicotinamide adenine dinucleotide phosphate-oxidase activation independently of COX inhibition, preventing oxidative damage in the brain (40). These results indicate that enhancement of anti-oxidation function, potentially via PPAR $\gamma$ activation, contributes to the renal protection of ibuprofen in type 1 diabetes.

Although in the current study ibuprofen or pioglitazone treatment did not influence the nFBG level and the body weight of diabetic rats, ibuprofen and pioglitazone showed significant attenuation on renal function and morphological changes in diabetic rats. There have been similar studies regarding these effects of PPAR $\gamma$ agonist; in experimental models of diabetes, PPAR $\gamma$ agonists have been shown to attenuate renal damage and reduce albuminuria, independent to blood glucose lowering (41). Previous studies have indicated that a low dose of pioglitazone ameliorated renal fibrosis and preserved renal function in an animal model of metabolic 
A
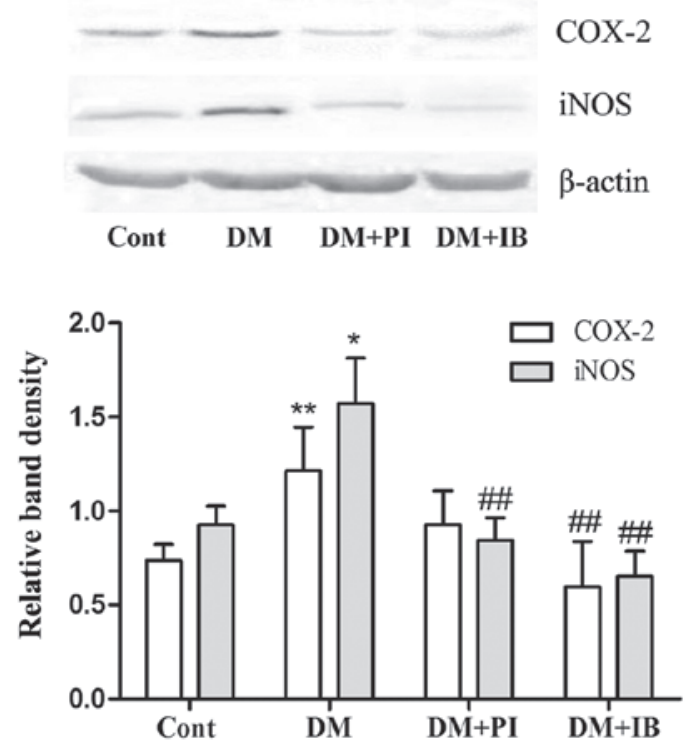

B

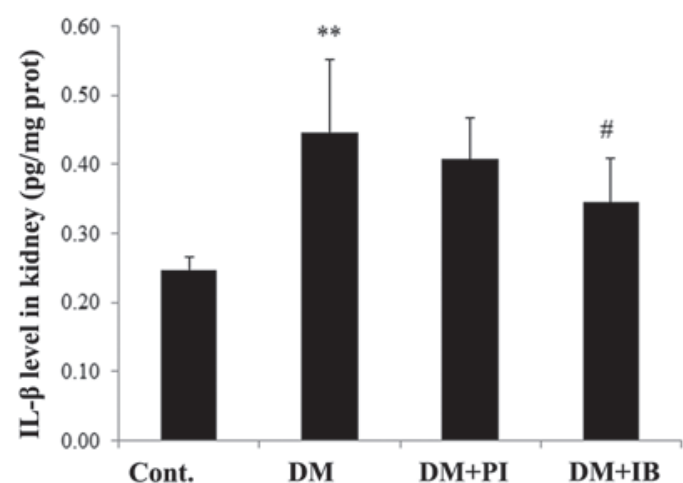

C

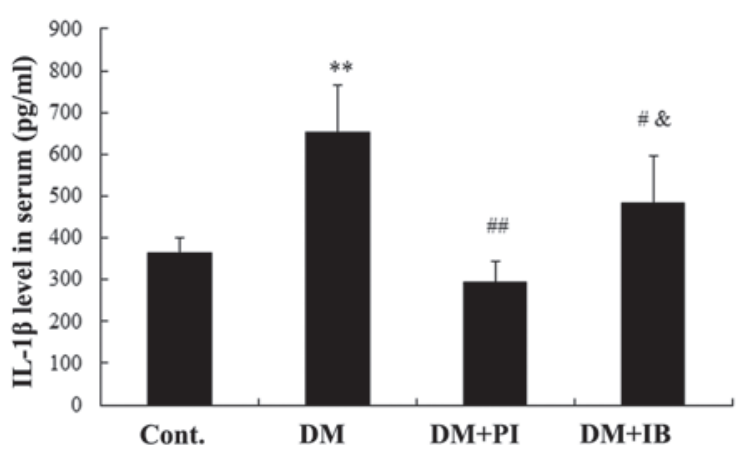

Figure 5. Effects of IB on protein (prot) expression of COX-2 and iNOS in (A) rat kidneys, and IL-1 levels in rat (B) kidneys and (C) serum. COX-2 and iNOS protein expression levels were assayed by western blotting. The IL-1 level was measured by enzyme linked immunosorbent assay. The Cont., $\mathrm{DM}, \mathrm{DM}+\mathrm{PI}$, and DM + IB groups represent healthy rats, diabetic rats, and diabetic rats treated with PI $(25 \mathrm{mg} / \mathrm{kg})$ and IB $(40 \mathrm{mg} / \mathrm{kg})$, respectively. Data are presented as means \pm standard deviation; $n=4$ (COX-2 and iNOS); $\mathrm{n}=6-8$ (IL-1). ${ }^{*} \mathrm{P}<0.05$ and ${ }^{* *} \mathrm{P}<0.01$ vs. Cont.; ${ }^{*} \mathrm{P}<0.05$ and ${ }^{\# \#} \mathrm{P}<0.01$ vs. $\mathrm{DM}$; ${ }^{\&} \mathrm{P}<0.05$ vs. DM + PI group. COX-2, cyclooxygenase 2; iNOs, inducible nitric oxide synthase; IL-1, interleukin-1; Cont., control; DM, diabetic group; PI, pioglitazone; IB, ibuprofen.

syndrome, independently of hyperglycemic control or effects on body weight $(12,42)$. A recent review demonstrated that PPAR $\gamma$ agonist-mediated renal protection resulted from numerous other efficacies beyond the glucose lowering effect (43). Together, these findings further confirmed the key role of anti-inflammatory and anti-oxidative actions of
A

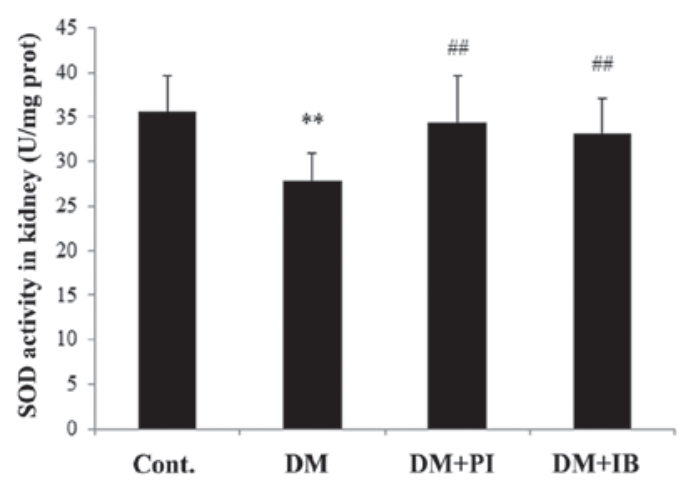

B

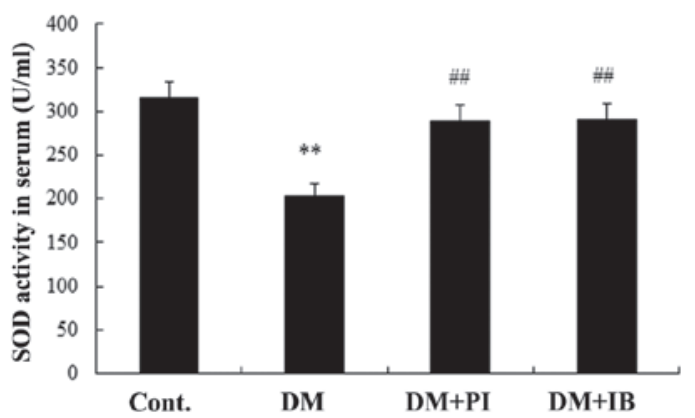

Figure 6. Effects of IB on SOD activity in rat (A) kidneys and (B) serum. SOD activity was measured using xanthine oxidase (the hydroxylamine method). The Cont., DM, DM + PI, and DM + IB groups represent healthy rats, diabetic rats, and diabetic rats treated with PI $(25 \mathrm{mg} / \mathrm{kg})$ and $\mathrm{IB}$ $(40 \mathrm{mg} / \mathrm{kg})$, respectively. Data are presented as means \pm standard deviation; $\mathrm{n}=7-8 .{ }^{* *} \mathrm{P}<0.01$ vs. Cont.; ${ }^{\# \#} \mathrm{P}<0.01$ vs. DM. SOD, superoxide dismutase; Cont., control; DM, diabetic group; PI, pioglitazone; IB, ibuprofen.

A

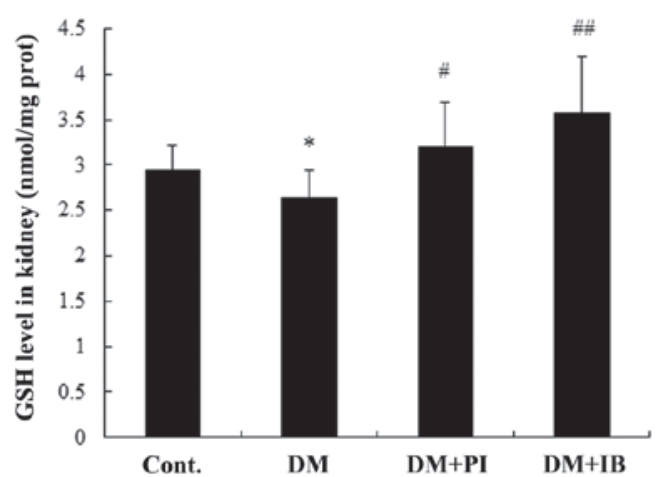

B

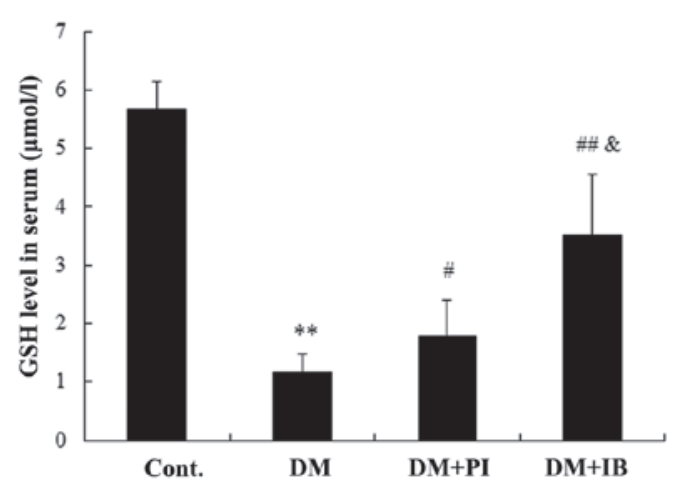

Figure 7. Effects of IB on reduced GSH levels in rat (A) kidneys and (B) serum. The GSH level was measured by spectrophotometry. Cont., DM, DM + PI, and DM + IB groups represent healthy rats, diabetic rats, and diabetic rats treated with PI $(25 \mathrm{mg} / \mathrm{kg})$ and IB $(40 \mathrm{mg} / \mathrm{kg})$, respectively. Data are presented as means \pm standard deviation; $\mathrm{n}=7-8$. ${ }^{*} \mathrm{P}<0.05$ and ${ }^{* *} \mathrm{P}<0.01$ vs. Cont.; ${ }^{\#} \mathrm{P}<0.05$ and $^{\# \#} \mathrm{P}<0.01$ vs. DM; ${ }^{\&} \mathrm{P}<0.05$ vs. DM + PI. GSH, glutathione; Cont., control; DM, diabetic group; PI, pioglitazone; IB, ibuprofen. 
ibuprofen via PPAR $\gamma$ activation in the prevention and treatment of type 1 diabetes-induced nephropathy.

In conclusion, the present study demonstrated that ibuprofen markedly attenuated the functional and morphological changes in the kidney of rats with STZ-induced type 1 diabetes, which was realized by anti-inflammatory and anti-oxidative action, potentially via COX-2 suppression and PPAR $\gamma$ activation, suggesting that ibuprofen may serve as a multi-target therapeutic agent for DN.

\section{Acknowledgements}

The present study was funded by the China Postdoctoral Science Foundation (grant no. 201150M1576), the Zhen Xing Project of XZMC, and a project funded through the Priority Academic Program Development of Jiangsu Higher Education Institutions.

\section{References}

1. Biesenbach G: Highest mortality during the last year before and the first year after start of dialysis treatment in type 2 diabetic patients with nephropathy. Curr Diabetes Rev 3: 123-126, 2007.

2. Schernthaner G: Kidney disease in diabetology: Lessons from 2007. Nephrol Dial Transplant 23: 1112-1115, 2008.

3. Simonson MS: Phenotypic transitions and fibrosis in diabetic nephropathy. Kidney Int 71: 846-854, 2007.

4. Qian Y, Feldman E, Pennathur S, Kretzler M and Brosius FC III: From fibrosis to sclerosis: Mechanisms of glomerulosclerosis in diabetic nephropathy. Diabetes 57: 1439-1445, 2008.

5. Swaminathan S and Shah SV: Novel approaches targeted toward oxidative stress for the treatment of chronic kidney disease. Curr Opin Nephrol Hypertens 17: 143-148, 2008.

6. Ohga S, Shikata K, Yozai K, Okada S, Ogawa D, Usui H, Wada J, Shikata Y and Makino H: Thiazolidinedione ameliorates renal injury in experimental diabetic rats through anti-inflammatory effects mediated by inhibition of NF-kappaB activation. Am J Physiol Renal Physiol 292: F1141-1150, 2007.

7. Willson TM, Lambert MH and Kliewer SA: Peroxisome proliferator-activated receptor gamma and metabolic disease. Annu Rev Biochem 70: 341-367, 2001.

8. Baylis C, Atzpodien EA, Freshr G and Engels K: Peroxisome proliferator-activated receptor [gamma] agonist provides superior renal protection versus angiotensin-converting enzyme inhibition in a rat model of type 2 diabetes with obesity. J Pharmacol Exp Ther 307: 854-860, 2003.

9. Yang J, Zhang D, Li J, Zhang X, Fan F and Guan Y: Role of PPARgamma in renoprotection in Type 2 diabetes: Molecular mechanisms and therapeutic potential. Clin Sci (Lond) 116 17-26, 2009.

10. Yang J, Zhou Y and Guan Y: PPARgamma as a therapeutic target in diabetic nephropathy and other renal diseases. Curr Opin Nephrol Hypertens 21: 97-105, 2012.

11. Hu W, Yu Q, Zhang J and Liu D: Rosiglitazone ameliorates diabetic nephropathy by reducing the expression of Chemerin and ChemR23 in the kidney of streptozotocin-induced diabetic rats. Inflammation 35: 1287-1293, 2012.

12. Arora MK, Reddy K and Balakumar P: The low dose combination of fenofibrate and rosiglitazone halts the progression of diabetes-induced experimental nephropathy. Eur J Pharmacol 636: 137-144, 2010.

13. Groop PH, Thomas MC, Moran JL, Wadèn J, Thorn LM, Mäkinen VP, Rosengård-Bärlund M, Saraheimo M, Hietala K, Heikkilä $\mathrm{O}$, et al: The presence and severity of chronic kidney disease predicts all-cause mortality in type 1 diabetes. Diabetes 58: 1651-1658, 2009.

14. Fogo AB: PPARgamma and chronic kidney disease. Pediatr Nephrol 26: 347-351, 2011.

15. Iglesias $P$ and Díez JJ: Peroxisome proliferator-activated receptor gamma agonists in renal disease. Eur J Endocrinol 154: 613-621, 2006.

16. Elmarakby AA and Sullivan JC: Relationship between oxidative stress and inflammatory cytokines in diabetic nephropathy. Cardiovasc Ther 30: 49-59, 2012.
17. Gumieniczek A: Effect of the new thiazolidinedione-pioglitazone on the development of oxidative stress in liver and kidney of diabetic rabbits. Life Sci 74: 553-562, 2003.

18. Lakshmanan AP, Watanabe K, Thandavarayan RA, Sari FR, Harima M, Giridharan VV, Soetikno V, Kodama M and Aizawa Y: Telmisartan attenuates oxidative stress and renal fibrosis in streptozotocin induced diabetic mice with the alteration of angiotensin-(1-7) mas receptor expression associated with its PPAR-gamma agonist action. Free Radic Res 45: 575-584, 2011.

19. Matsui T, Yamagishi S, Ueda S, Nakamura K, Imaizumi T, Takeuchi $\mathrm{M}$ and Inoue $\mathrm{H}$ : Telmisartan, an angiotensin II type 1 receptor blocker, inhibits advanced glycation end-product (AGE)-induced monocyte chemoattractant protein-1 expression in mesangial cells through downregulation of receptor for AGEs via peroxisome proliferator-activated receptor-gamma activation. J Int Med Res 35: 482-489, 2007.

20. Matsui T, Yamagishi S, Takeuchi M, Ueda S, Fukami K and Okuda S: Nifedipine inhibits advanced glycation end products (AGEs) and their receptor (RAGE) interaction-mediated proximal tubular cell injury via peroxisome proliferator-activated receptor-gamma activation. Biochem Biophys Res Commun 398: 326-330, 2010.

21. Mohamed R, Jayakumar C, Ranganathan PV, Ganapathy V and Ramesh G: Kidney proximal tubular epithelial-specific overexpression of netrin-1 suppresses inflammation and albuminuria through suppression of COX-2-mediated PGE2 production in streptozotocin-induced diabetic mice. Am J Pathol 181: 1991-2002, 2012.

22. Jaradat MS, Wongsud B, Phornchirasilp S, Rangwala SM, Shams G, Sutton M, Romstedt KJ, Noonan DJ and Feller DR: Activation of peroxisome proliferator-activated receptor isoforms and inhibition of prostaglandin $\mathrm{H}(2)$ synthases by ibuprofen, naproxen and indomethacin. Biochem Pharmacol 62: 1587-1595, 2001.

23. Couto M: Laboratory guidelines for animal care. Methods Mol Biol 770: 579-599, 2011.

24. Haidara MA, Mikhailidis DP, Rateb MA, Ahmed ZA, Yassin HZ, Ibrahim IM and Rashed LA: Evaluation of the effect of oxidative stress and vitamin E supplementation on renal function in rats with streptozotocin-induced Type 1 diabetes. J Diabetes Complications 23: 130-136, 2009.

25. Wybenga DR, Di Giorgio J and Pileggi VJ: Manual and automated methods for urea nitrogen measurement in whole serum. Clin Chem 17: 891-895, 1971.

26. Liu YW, Zhu X, Zhang L, Lu Q, Wang JY, Zhang F, Guo H, Yin JL and Yin XX: Up-regulation of glyoxalase 1 by mangiferin prevents diabetic nephropathy progression in streptozotocin-induced diabetic rats. Eur J Pharmacol 721: 355-364, 2013.

27. Liu YW, Zhu X, Yang QQ, Lu Q, Wang JY, Li HP, Wei YQ, Yin JL and Yin XX: Suppression of methylglyoxal hyperactivity by mangiferin can prevent diabetes-associated cognitive decline in rats. Psychopharmacology (Berl) 228: 585-594, 2013.

28. Chow FY, Nikolic-Paterson DJ, Atkins RC and Tesch GH: Macrophages in streptozotocin-induced diabetic nephropathy: Potential role in renal fibrosis. Nephrol Dial Transplant 19: 2987-2996, 2004.

29. Nguyen D, Ping F, Mu W, Hill P, Atkins RC and Chadban SJ: Macrophage accumulation in human progressive diabetic nephropathy. Nephrology (Carlton) 11: 226-231, 2006.

30. Rivero A, Mora C, Muros M, García J, Herrera H and Navarro-González JF: Pathogenic perspectives for the role of inflammation in diabetic nephropathy. Clin Sci (Lond) 116: 479-492, 2009

31. Tuttle KR: Linking metabolism and immunology: Diabetic nephropathy is an inflammatory disease. J Am Soc Nephrol 16: 1537-1538, 2005.

32. Yozai K, Shikata K, Sasaki M, Tone A, Ohga S, Usui H, Okada S, Wada J, Nagase R, Ogawa D, et al: Methotrexate prevents renal injury in experimental diabetic rats via anti-inflammatory actions. J Am Soc Nephrol 16: 3326-3338, 2005.

33. Nasrallah R, Robertson SJ and Hébert RL: Chronic COX inhibition reduces diabetes-induced hyperfiltration, proteinuria and renal pathological markers in 36-week B6-Ins2(Akita) mice. Am J Nephrol 30: 346-353, 2009.

34. Elmarakby AA, Abdelsayed R, Yao Liu J and Mozaffari MS: Inflammatory cytokines as predictive markers for early detection and progression of diabetic nephropathy. EPMA J 1: 117-129, 2010. 
35. Kawai T, Masaki T, Doi S, Arakawa T, Yokoyama Y, Doi T, Kohno N and Yorioka N: PPAR-gamma agonist attenuates renal interstitial fibrosis and inflammation through reduction of TGF-beta. Lab Invest 89: 47-58, 2009.

36. Ha $\mathrm{H}$ and Lee HB: Oxidative stress in diabetic nephropathy: Basic and clinical information. Curr Diab Rep 1: 282-287, 2001.

37. Forbes JM, Coughlan MT and Cooper ME: Oxidative stress as a major culprit in kidney disease in diabetes. Diabetes 57 1446-1454, 2008.

38. Brownlee M: The pathobiology of diabetic complications: A unifying mechanism. Diabetes 54: 1615-1625, 2005.

39. Gumieniczek A: Effects of pioglitazone on hyperglycemia-induced alterations in antioxidative system in tissues of alloxan-treated diabetic animals. Exp Toxicol Pathol 56: 321-326, 2005.
40. Wilkinson BL, Cramer PE, Varvel NH, Reed-Geaghan E, Jiang Q, Szabo A, Herrup K, Lamb BT and Landreth GE: Ibuprofen attenuates oxidative damage through NOX2 inhibition in Alzheimer's disease. Neurobiol Aging 33: 197.e21-32, 2012.

41. Isshiki K, Haneda M, Koya D, Maeda S, Sugimoto T and Kikkawa R: Thiazolidinedione compounds ameliorate glomerular dysfunction independent of their insulin-sensitizing action in diabetic rats. Diabetes 49: 1022-1032, 2000.

42. Toblli JE, Ferrini MG, Cao G, Vernet D, Angerosa M and Gonzalez-Cadavid NF: Antifibrotic effects of pioglitazone on the kidney in a rat model of type 2 diabetes mellitus. Nephrol Dial Transplant 24: 2384-2391,2009.

43. Sugawara A, Uruno A, Kudo M, Matsuda K, Yang CW and Ito S: PPAR $\gamma$ agonist beyond glucose lowering effect. Korean J Intern Med 26: 19-24, 2011. 